\title{
Metabolomics combined with network pharmacology exploration reveals the modulatory properties of Astragali Radix extract in the treatment of liver fibrosis
}

Dan Wang ${ }^{1,2}$, Ruisheng Li $i^{3}$, Shizhang Wei ${ }^{1,2}$, Sijia Gao ${ }^{1,2}$, Zhuo Xu ${ }^{1,2}$, Honghong Liu ${ }^{4}$, Ruilin Wang ${ }^{5}$, Haotian Li ${ }^{2}$, Huadan Cai ${ }^{2}$, Jian Wang ${ }^{1 *}$ (D) and Yanling Zhao ${ }^{2^{*}}$

\begin{abstract}
Background: Astragali Radix (AR) is widely-used for improving liver fibrosis, but, the mechanism of action has not been systematically explained. This study aims to investigate the mechanism of AR intervention in liver fibrosis based on comprehensive metabolomics combined with network pharmacology approach.

Materials and methods: UPLC-Q-TOF/MS based metabolomics technique was used to explore the specific metabolites and possible pathways of AR affecting the pathological process of liver fibrosis. Network pharmacology analysis was introduced to explore the key targets of AR regarding the mechanisms on liver fibrosis.

Results: AR significantly reduced the levels of ALT, AST and AKP in serum, and improved pathological characteristics. Metabolomics analysis showed that the therapeutic effect of AR was mainly related to the regulation of nine metabolites, including sphingosine, 6-keto-prostaglandin F1a, LysoPC (O-18:0), 3-dehydrosphinganine, 5,6-epoxy-8,11,14eicosatrienoic acid, leukotriene C4, taurochenodesoxycholic acid, LysoPC (18:1 (9Z)) and 2-acetyl-1-alkyl-sn-glycero3-phosphocholine. Pathway analysis indicated that the treatment of AR on liver fibrosis was related to arachidonic acid metabolism, ether lipid metabolism, sphingolipid metabolism, glycerophospholipid metabolism and primary bile acid biosynthesis. Validation of the key targets by network pharmacology analysis of potential metabolic markers showed that AR significantly down-regulated the expression of CYP1B1 and up-regulated the expression of CYP1A2 and PCYT1A.

Conclusion: Metabolomics combined with network pharmacology was used for the first time to clarify that the treatment of AR on liver fibrosis, which is related to the regulation of arachidonic acid metabolism and ether lipid metabolism by modulating the expression of CYP1A2, CYP1B1 and PCYT1A. And the integrated approach can provide new strategies and ideas for the study of molecular mechanisms of traditional Chinese medicines in the treatment of liver fibrosis.
\end{abstract}

Keywords: Astragali Radix, Liver fibrosis, Metabolomics, Network pharmacology, Modulatory properties, Molecular mechanisms

\footnotetext{
*Correspondence: jianwang08@163.com; zhaoyl2855@126.com

1 Provincial and State Key Laboratory Breeding Base of System Research,

Development and Utilization of Chinese Herbal Medicine Resources,

College of Pharmacy, Chengdu University of Traditional Chinese

Medicine, Chengdu 611137, China

${ }^{2}$ Department of Pharmacy, The Fifth Medical Center of PLA General

Hospital, Beijing 100039, China

Full list of author information is available at the end of the article
}

(c) The Author(s) 2019. This article is distributed under the terms of the Creative Commons Attribution 4.0 International License (http://creativecommons.org/licenses/by/4.0/), which permits unrestricted use, distribution, and reproduction in any medium, provided you give appropriate credit to the original author(s) and the source, provide a link to the Creative Commons license, and indicate if changes were made. The Creative Commons Public Domain Dedication waiver (http://creativecommons.org/ publicdomain/zero/1.0/) applies to the data made available in this article, unless otherwise stated. 


\section{Background}

As a worldwide clinical problem, liver fibrosis is a wound healing process with recurrent chronic liver damage [1]. It can accelerate the development of chronic liver disease by destroying normal liver parenchyma, eventually leading to cirrhosis, liver failure and even primary liver cancer [2]. Although the pathogenesis of liver fibrosis, such as inflammatory response, hepatic stellate cell activation and extracellular matrix formation, has been widely recognized, it has not got an effective and powerful treatment $[3,4]$. Therefore, it is necessary to develop an effective treatment tool for liver fibrosis with high efficiency, low side effects and multiple targets.

Astragali Radix (AR), is a worldwide used traditional Chinese medicine (TCM) is the dried root of Astragali radix membranaceus (Fisch.) Bge. or Astragali radix membranaceus (Fisch.) Bge. var. mongholicus (Bge) Hsiao [5, 6]. In traditional Chinese formula, AR is often used for the treatment of liver fibrosis [6, 7]. Fundamental studies have exhibited the anti-hepatic fibrosis effects of AR by inhibiting TGF- $\beta /$ Smads signaling $[8,9]$. In addition, $A R$ and its active ingredients have obvious protective effects on cholestasis [8], carbon tetrachloride [10], dimethyl nitrosamine [8], acetaminophen [11] and ethanol [12] induced liver injury. Moreover, studies have shown that AR is safe without obvious toxicity, side effects or genotoxicity [13, 14]. Therefore, AR exhibits significant advantages for the treatment of liver fibrosis.

Metabolomics can characterize the dynamic changes of metabolites throughout the biological system providing a powerful platform for discovering new biomarkers and biochemical pathways [15, 16], improving diagnosis [17], treatment and prediction [18, 19] in complex systems. Although some studies have analyzed the metabolites of $A R$ in vivo, only the changes of endogenous metabolites of AR from different habitats in normal mice for quality evaluation were compared $[5,20]$. The analysis of metabolite changes of AR in diseased mice is currently lacking. In addition, network pharmacology has become a powerful tool for studying complex diseases to reveal the complex relationships between proteins, diseases and drugs [21]. This method helps to determine the main active ingredients of drugs and their role in the treatment of various diseases $[22,23]$. A combination of metabolomics and network pharmacology can link endogenous metabolites to disease targets, further to uncover the molecular mechanisms of TCM with multi-component and multitarget characteristics [24].

This study combined the UPLC-Q-TOF/MS serum metabolomics and network pharmacology techniques to systematically explain the modulatory properties of AR on liver fibrosis. Multivariate data analysis was used to screen potential metabolite makers and corresponding metabolic pathways to explore the function of AR. The feasibility of AR for the treatment of liver fibrosis was further confirmed by constructing a component-targetmetabolite network to identify the key targets on liver fibrosis (Fig. 1).

\section{Methods \\ Reagents}

Colchicine was got from XiShuangBanNa BanNa Pharmaceutical Co. (Yunnan, China). Carbon tetrachloride $\left(\mathrm{CCl}_{4}\right)$ was purchased from Tianjin Guangfu Chemical Research Institute (Tianjin, China). Alanine amino transferase (ALT), aspartate amino transferase (AST), alkaline phosphatase (AKP) detection kits were bought from Nanjing Jiancheng Bioengineering Institute (Nanjing, China). alpha smooth muscle actin $\alpha(\alpha-S M A)$ and transforming growth factor-beta (TGF- $\beta 1$ ) were bought from Cell Signaling Technology (United States). GAPDH was bought from ABclonal (China). Quercetin and nicotinic acid were bought from Chengdu Cloma Biological Co., Ltd (China).

\section{Preparation of AR water extract}

AR (the dried root of Astragali radix membranaceus (Fisch.) Bge.) was purchased from Beijing Lvye pharmaceutical co. (Beijing, China). Firstly, AR was extracted with boiling water $(1 / 10, w / v)$ for $2 \mathrm{~h}$. Then, it was extracted twice with boiling water $(1 / 8, w / v)$ for $1.5 \mathrm{~h}$ each time. The aqueous extract of AR was dried to power by freeze vacuum drying oven, and the final yield of powder was about $40.08 \%$. A Q-TOF LC/MS analysis was carried out to identify the main constituent in the tested extract (Additional file 1: Figure S1 and Table S1).

The active ingredients of AR extract were identified on Triple TOF 4600 high-resolution mass spectrometry system (AB SCIEX, USA), and $3 \mu \mathrm{L}$ of each sample was injected into ZORBAX Eclipse $\mathrm{C} 8$ analytical column (1.8 $\mu \mathrm{m}$ i.d., $2.1 \mathrm{~mm}$ i.d. $\times 100 \mathrm{~mm}$, Agilent Technologies, USA). The column temperature was maintained at $35{ }^{\circ} \mathrm{C}$. The flow rate was set as $0.25 \mathrm{~mL} / \mathrm{min}$. the mobile phase was solvent A (water with $0.1 \%$ formic acid) and solvent B (acetonitrile with $0.1 \%$ formic acid). The gradient was used as follows: a linear gradient of $0 \% \mathrm{~B}$ over initial-1.0 $\mathrm{min}, 0-5 \% \mathrm{~B}$ over $1.0-8.0 \mathrm{~min}, 5-55 \% \mathrm{~B}$ over 8.0-20.0 $\mathrm{min}, 55 \% \mathrm{~B}$ in $20.0-26.0 \mathrm{~min}$ and $55-5 \%$ B over 26.0-30.0 $\mathrm{min}$. The eluent was directly introduced into the mass spectrometer. The mass spectrometry conditions were as follows: the electrospray capillary voltage was $5.5 \mathrm{kV}$ in positive ionization mode and $4.5 \mathrm{kV}$ in negative ionization mode. The Gas1 and Gas2 were 55 Psi, the gas temperature was $600{ }^{\circ} \mathrm{C}$, and the collision voltage was $40 \mathrm{~V}$ in the positive and negative ionization mode. The full scan mode was adopted and with the aid 


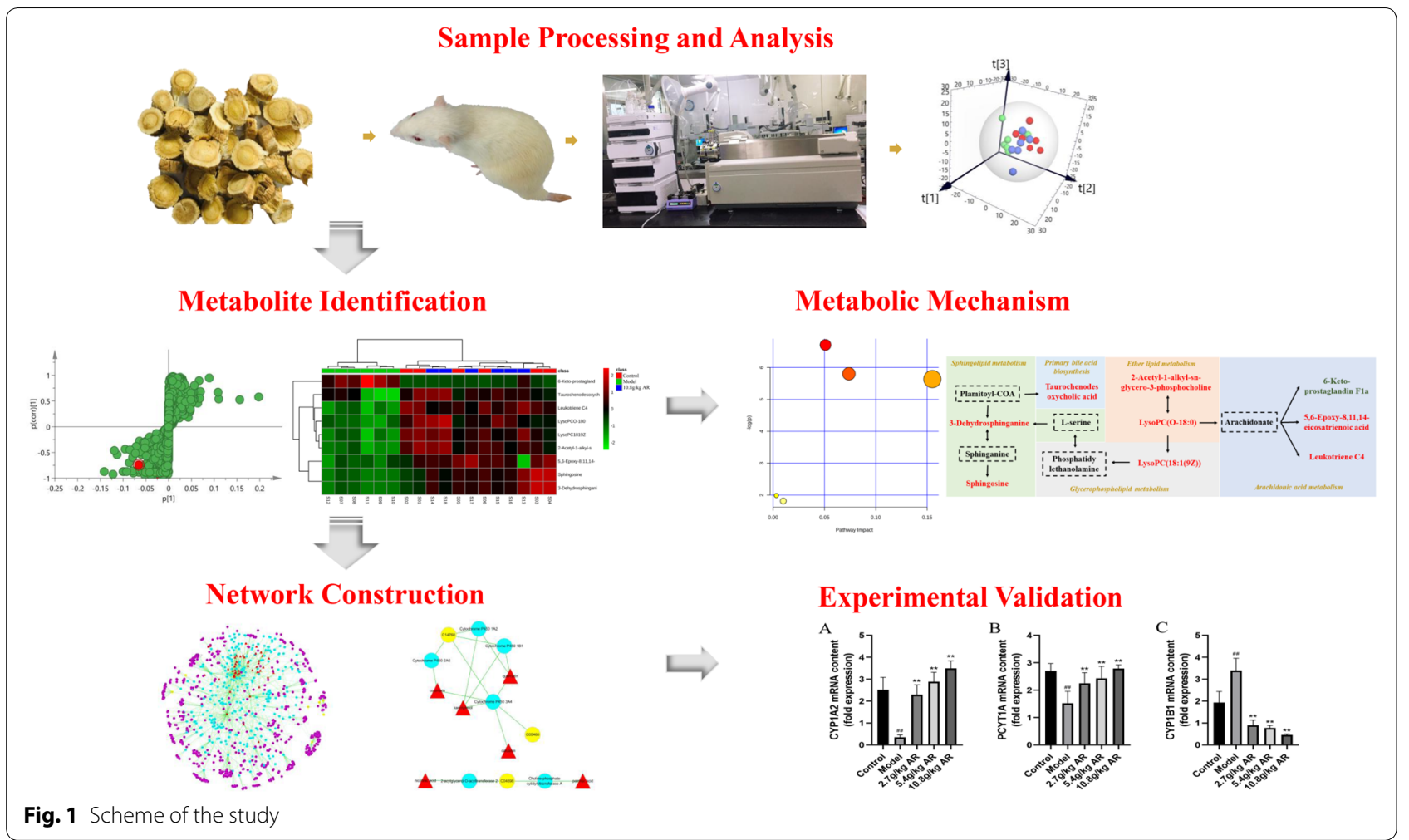

of information correlation acquisition and background subtraction technology. The first scanning frequency was $0.2 \mathrm{~s}$ and the second scan frequency was $0.12 \mathrm{~s}$.

\section{Animal experiments and sample collection}

180-200 g male Sprague-Dawley rats were offered by the China food and drug certification research institute (Permission NO. SCXK(Jing)-2014-0013) and housed with same feeding environment (temperature: $25{ }^{\circ} \mathrm{C} \pm 2{ }^{\circ} \mathrm{C}$, humidity: $55 \% \pm 5 \% 12: 12 \mathrm{~h}$ light: dark cycle) in the Central Animal Laboratory of The Fifth Medical Center of PLA General Hospital.

Thirty-six rats were randomly divided into six groups. The control group was only given with olive oil and the other five groups were given with intraperitoneal administration of olive oil $(1: 1, v / v)$ dissolved $\mathrm{CCl}_{4}(2 \mathrm{~mL} / \mathrm{kg})$ twice a week for 8 weeks. Then AR $(10.8,5.4,2.7 \mathrm{~g} / \mathrm{kg} /$ day) and colchicine $(0.2 \mathrm{mg} / \mathrm{kg} /$ day, as a positive control group) were administered by intragastric administration for 6 weeks, except for the control and model groups, and $\mathrm{CCl}_{4}$ was given intraperitoneally at the same time.

After $12 \mathrm{~h}$ of the last treatment, the rats were sacrificed and liver and blood were collected. The blood was centrifuged at $3500 \mathrm{rpm}$ for $15 \mathrm{~min}$ to separate the serum without hemolysis. Serum and liver tissue were stored at $-80{ }^{\circ} \mathrm{C}$ for the biochemical parameters and metabolomics analysis. All animal experiments were approved by the Ethical Committee of Fifth Medical Center of PLA General Hospital of China.

Serum levels of ALT, AST and AKP were tested according to the instruction of kits. Liver tissue was fixed in $10 \%$ neutral buffered formalin for $24 \mathrm{~h}$. All fixed livers were embedded in paraffin, and then were cut into sections (about $4-5 \mu \mathrm{m}$ thick) by using a microtome. Hematoxylin-eosin (HE) and Masson staining were used for highlighting the liver damage and collagen deposition, respectively.

\section{Immunofluorescence and immunohistochemistry analysis}

To measure immunofluorescence, liver tissues were sliced into $14 \mu \mathrm{m}$ thick sections and then blocked with blocking buffer containing $0.01 \mathrm{M}$ phosphate buffered saline (PBS), $0.1 \%$ Triton $\mathrm{X}-100$ and $5 \%$ normal goat serum solution for $60 \mathrm{~min}$. Subsequently, the sections were incubated with primary antibody [TGF- $\beta 1$ (1:100)]. The sections were washed and incubated with an antirabbit Alexa Fluor 488-conjugated IgG secondary antibody. 4,6-diamidino-2-phenylindole counterstaining was used to stain the nuclei. The sections were cover-slipped with fluorescent mounting medium.

To measure immunohistochemical, liver tissues were immersed in $2 \% \mathrm{H}_{2} \mathrm{O}_{2}$ for $25 \mathrm{~min}$ at room temperature and then blocked with $5 \%$ rabbit serum for $30 \mathrm{~min}$. Then, 
the primary antibody [ $\alpha$-SMA (1:100)] was added for incubation at $4{ }^{\circ} \mathrm{C}$ overnight. After washing with PBS, the liver sections were incubated with horseradish peroxidase-conjugated secondary antibody for $50 \mathrm{~min}$ at room temperature. Liver sections were immersed in diaminobenzidine for $3 \mathrm{~min}$ and then stained with ethanol dehydrated hematoxylin. The stained areas of the sections were observed under an optical microscope of $200 \mathrm{~nm}$.

\section{Western blotting}

Liver tissue (80 $\mathrm{mg}$ ) was homogenized and lysed in iceCold lysis buffer with PMSF and protein phosphatase inhibitor mixture, and then centrifuged at $12,000 \times g$ and $4{ }^{\circ} \mathrm{C}$ for a $10 \mathrm{~min}$. The supernatant was western blotted with 10\% SDS-PAGE and the blot was transferred to the polyvinylidene fluoride membrane. Subsequently, it was incubated with $5 \%$ skim milk powder in blocking buffer for $2 \mathrm{~h}$. And polyvinylidene fluoride membrane incubated with $\alpha$-SMA $(1: 1000)$ and GAPDH $(1: 2000)$ primary antibodies at $4{ }^{\circ} \mathrm{C}$ with gentle shaking overnight. Finally, after incubation with the secondary antibody for $1 \mathrm{~h}$, the membrane was washed 3 times with TBST for 5 min each time and the immunoreactivity bands were detected by chemiluminescence detection kit.

\section{Sample preparation and UPLC-Q-TOF/MS assay}

$600 \mu \mathrm{L}$ of methanol was added to $200 \mu \mathrm{L}$ of serum and was mixed. The sample was allowed to stand at $4{ }^{\circ} \mathrm{C}$ for $20 \mathrm{~min}$ and then centrifuged at $12,000 \mathrm{rpm}$ for $10 \mathrm{~min}$. Finally, the supernatant was absorbed and filtered through $0.22 \mu \mathrm{m}$ micropore filter. The filtrate was collected for analysis. For the quality control (QC) samples, taken $20 \mu \mathrm{L}$ from each prepare sample extract and mix, used the rest of the samples for Q-TOF LC/MS test.

An Agilent 6550 iFunnel Q-TOF LC/MS (Agilent Technologies, USA) system was used for serum metabolic spectrum analysis. A $4 \mu \mathrm{L}$ aliquot of each sample was injected into the system on a ZORBOX RRHD C18 analytical column (2.1 mm i.d. $\times 100 \mathrm{~mm}, 1.8 \mu$ mi.d., Agilent Technologies, USA) for sample separation at $30^{\circ} \mathrm{C}$. Solvent $\mathrm{A}$ (water containing $0.1 \%$ formic acid) and solvent $\mathrm{B}$ (acetonitrile containing $0.1 \%$ formic acid) were used as the mobile phase for a linear gradient separation at a flow rate of $0.30 \mathrm{~mL} / \mathrm{min}$ for $25 \mathrm{~min}$ a linear gradient of $100 \%$ A over 0-1.0 min, 100-60\% A over 1.0-9.0 min, 60-10\% A over $9.0-19.0 \mathrm{~min}, 10-0 \%$ A over $19.0-21.0 \mathrm{~min}, 100 \%$ B over 21.0-25.0 min.

Both positive and negative mode electrospray ionization sources (ESI) were used. The electrospray source parameters are set as follows: electrospray capillary voltage is $3.5 \mathrm{kV}$ in negative ionization mode and $4 \mathrm{kV}$ in positive ionization mode, mass range is from $\mathrm{m} / z 50$ to
1200 , gas temperature is $225{ }^{\circ} \mathrm{C}$, gas flow rate is $13 \mathrm{~L} /$ $\mathrm{min}$, nebulizer is set to $20 \mathrm{psi}$, sheath gas temperature is $275^{\circ} \mathrm{C}$, sheath gas flow is $12 \mathrm{~L} / \mathrm{min}$, and nozzle voltage is $2000 \mathrm{~V}$ in both negative and positive modes.

\section{Data extraction and multivariate analysis}

MassHunter Profinder software (Agilent, California, United States) was used to extract sample data and perform peak detection and alignment. The full scan mode is applied to the mass range of $m / z \quad 80-1000$ and sets the initial and final retention times for data collection. Data was normalized using MetaboAnalyst 4.0 and then analyzed by principal component analysis (PCA) and orthogonal-partial least squares-discriminant analysis (OPLS-DA) using SIMCA-P 14.1 software (Umetrics, Umea, Sweden).

\section{Biomarkers identification and pathway enrichment analysis}

Biomarkers were discovered by screening for metabolic differences. Differential metabolite satisfying the conditions (VIP $>1.0, \mid \mathrm{p}$ (corr) $\mid \geq 0.58$ and $P<0.05$ in ANOVA) were used as potential biomarkers in the OPLS-DA analysis [24]. Metabolites (molecular weight error $<20 \mathrm{ppm}$ ) were identified based on precise molecular weight in the Human Metabolome Database (HMDB) and Metlin database. The identified compounds were resubmitted to MetaboAnalyst 4.0 to analyze their signaling pathways.

\section{Identification of drug targets and network construction}

The chemical components of AR (oral bioavailability $(\mathrm{OB}) \geq 30 \%$ and a drug-likeness $(\mathrm{DL})>0.18$ ) were collected in the TCMSP database. And the MBROLE 2.0 database was used to collect the protein targets for potential metabolites. UniProt ID was used to replace different types of protein IDs, and then a metabolictarget-component interaction network was established through protein interaction (PPI) information. Finally, the network was visualized and analyzed using Cytoscape 3.6.1 software.

\section{Real-time polymerase chain reaction (RT-PCR) detection}

Total RNA was extracted from liver tissue by trizol reagent and RNA (2 $\mu$ g) was transcribed into cDNA by PrimerScript RT regent kit. The cDNA was subsequently subjected to PCR amplification by ABI Step One Plus. Data analysis was performed by $2^{-\Delta \Delta C T}$ method. The primers are listed in Table 1.

\section{Statistical analysis}

Data were analyzed by one-way ANOVA and Duncan's multi-range test. The SPSS computer program was used 
Table 1 Primer sequences used for RT-PCR

\begin{tabular}{lll}
\hline Gene & Forward & Reverse \\
\hline CYP1A2 & GTGGTGGAATCGGTGGCT & $\begin{array}{c}\text { CTTGCTGCTCTTCACGAGGTT } \\
\text { GAG }\end{array}$ \\
CYP1B1 & CGAGAGTC & CTCGGCATCGTCGTGGTTGTAC \\
& TTGG & \\
PCYT1A & AGACGAGGTGGTGAGGAA & TGGAGATGCCTTCTGTCCTCT \\
& CGC & GTG \\
a-SMA & GGCCACTGCTGCTTCCTCTTC & TGCCCGCCGACTCCATTCC \\
TGF-B1 & ATGGTGGACCGCAACAACGC & CTGGCACTGCTTCCCGATGTC \\
GAPDH & GTCCATGCCATCACTGCCACTC & GATGACCTTGCCCACAGCCTTG \\
\hline
\end{tabular}

to analyze the mean of Windows. Results are expressed as mean \pm standard deviation (SD). $P<0.05$ was considered statistically significant.

\section{Results}

\section{AR reduced pathological damage in $\mathrm{CCl}_{4}$-induced liver} fibrosis

As shown in Fig. 2a-c, serum ALT, AST and AKP levels in the model group were significantly increased $(P<0.01)$ compared with that in the control group,

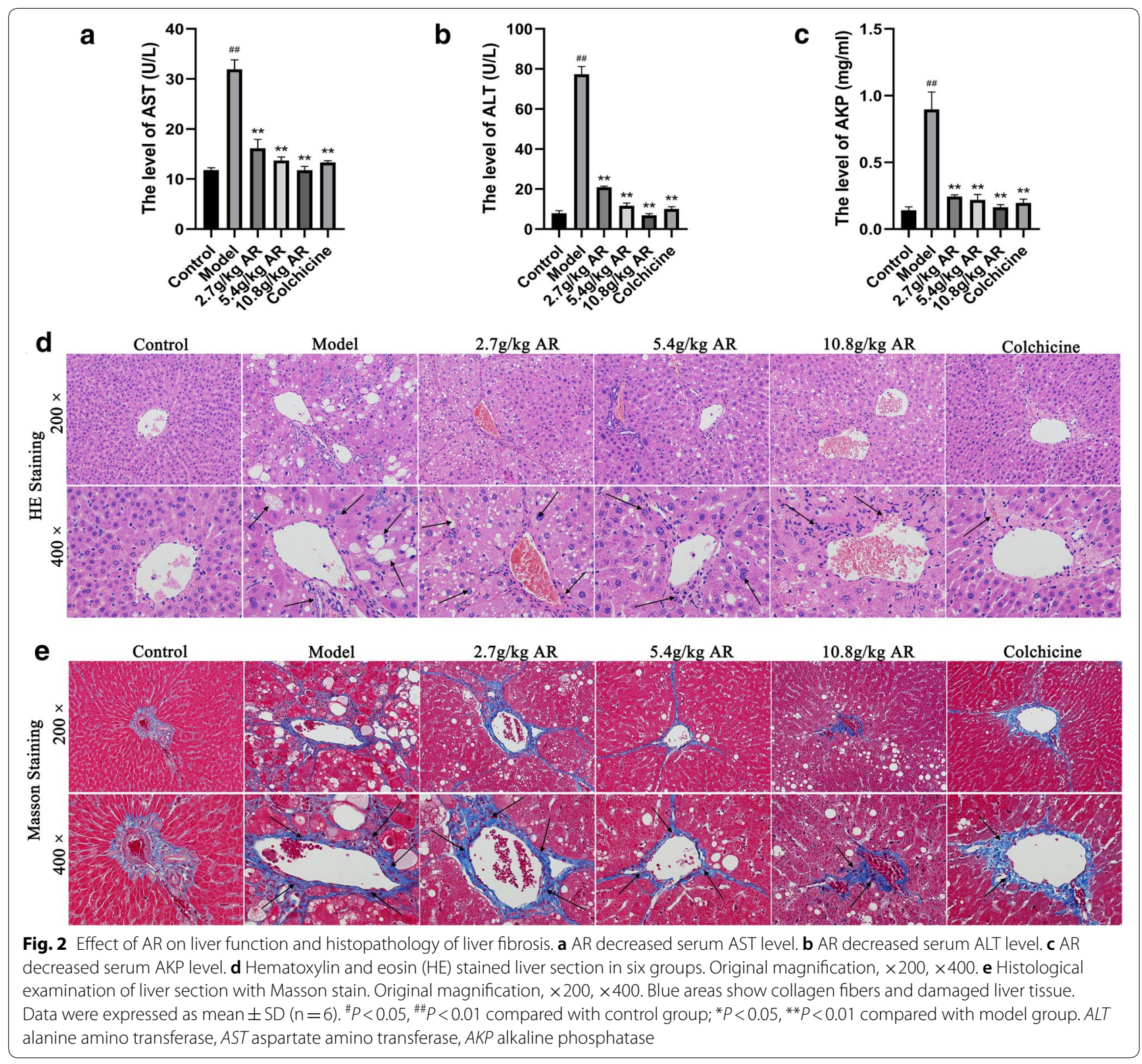


respectively. And serum ALT, AST and AKP concentrations were attenuated in a dose-dependent manner by AR. Compared with the model group, ALT, AKP and AST levels were most significantly reduced after administration of $10.8 \mathrm{~g} / \mathrm{kg}$ AR alone $(P<0.01)$. Histologic analysis was performed using HE staining and MASSON staining. As shown in Fig. 2d, the liver structure of rats in the control group was normal, and several histological features of the liver in $\mathrm{CCl}_{4}$-administration group included pericentral necrosis and fibrosis, vacuolar steatosis, inflammatory cell infiltration, and cytoplasmic degeneration. AR treatment of animals exposed to $\mathrm{CCl}_{4}$ can effectively improve liver necrosis, fibrosis, and reduce inflammatory infiltration, especially in $10.8 \mathrm{~g} / \mathrm{kg}$ AR group. In the liver section of MASSON staining, the blue area represented the deposited collagen. The results showed that a large amount of collagen was deposited around the hepatic sinus of the model group. And compared with model group, $10.8 \mathrm{~g} /$ $\mathrm{kg}$ AR could effectively reduce collagen deposition and improve liver fibrosis (Fig. 2e).

\section{AR reduced $\mathrm{CCl}_{4}$-induced liver fibrosis}

$\alpha$-SMA and TGF- $\beta 1$ are two key markers of $\mathrm{CCl}_{4}$-induced liver fibrosis. Western blot, immunohistochemistry and immunofluorescence were used to analyze the correlation between AR and liver fibrosis. As shown in Fig. 3a, western blotting showed that the expression of $\alpha$-SMA protein in the model group was significantly higher than that in the control group. And $\alpha$-SMA expression was decreased in a dose-dependent manner by AR. These results were consistent with the results observed via immunohistochemistry (Fig. 3b). Immunofluorescence showed that TGF- $\beta 1$ expression in model group increased significantly and the expression of TGF- $\beta 1$ was lower than that in the model group after administration of AR, especially in the $10.8 \mathrm{~g} / \mathrm{kg}$ AR group (Fig. 3c). Furthermore, the mRNA levels of $\alpha$-SMA and TGF- $\beta 1$ were detected by RT-PCR. The results of RT-PCR confirmed the above results again (Fig. $3 \mathrm{~d}, \mathrm{e}$ ). In conclusion, these results suggest that $\mathrm{AR}$ can reduce $\mathrm{CCl}_{4}$-induced liver fibrosis in a dose-dependent manner. Therefore, $10.8 \mathrm{~g} /$ $\mathrm{kg}$ of AR was selected as the optimal effective dose for a

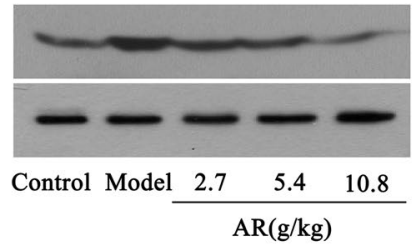

$\alpha$-SMA

GAPDH

$$
\text { Control Model } \begin{array}{llll}
2.7 & 5.4 & 10.8 \\
& \mathrm{AR}(\mathrm{g} / \mathrm{kg})
\end{array}
$$

b

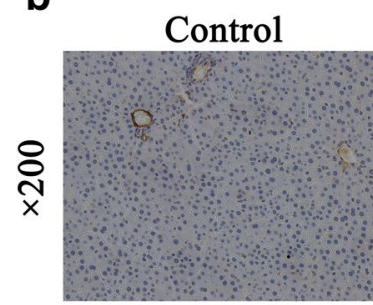

c

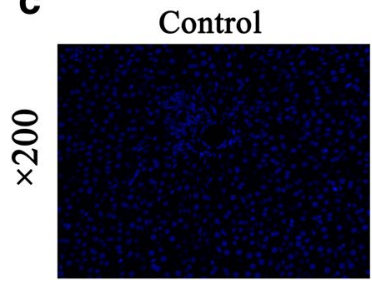

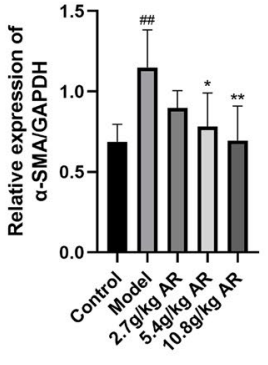

Model

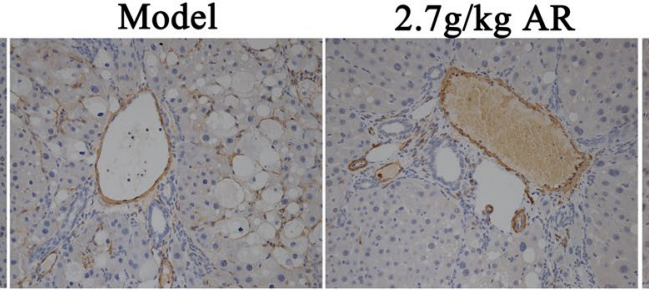

Model

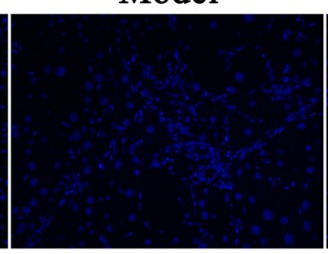

$2.7 \mathrm{~g} / \mathrm{kg} \mathrm{AR}$
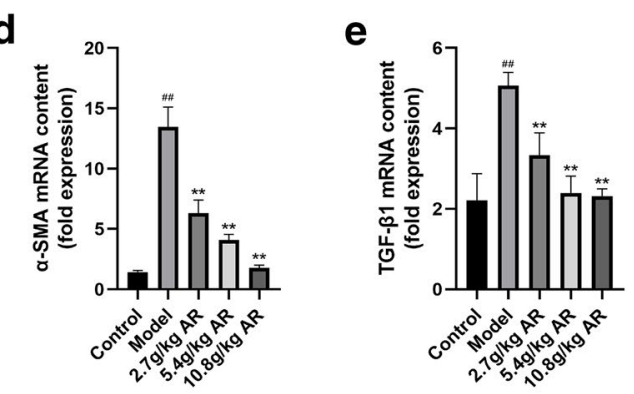

$5.4 \mathrm{~g} / \mathrm{kg} \mathrm{AR}$

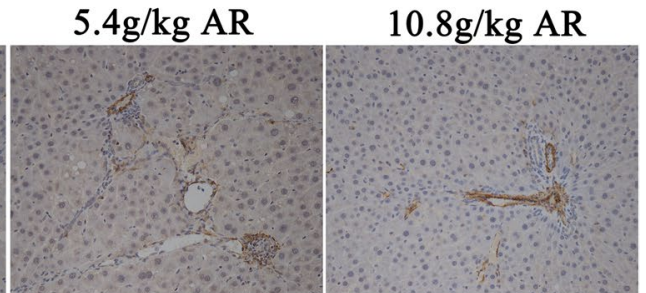

$5.4 \mathrm{~g} / \mathrm{kg} \mathrm{AR}$

$10.8 \mathrm{~g} / \mathrm{kg} \mathrm{AR}$

Fig. 3 AR alleviated $a-S M A$ and TGF- $\beta 1$ expression during liver fibrosis. a Western bolt analysis for a-SMA. b Immunohistochemical analysis for a-SMA. c Immunofluorescence analysis for TGF- $\beta 1$. d RT-PCR analysis for a-SMA. e RT-PCR analysis for TGF- $\beta 1$. Data were expressed as mean \pm SD $(\mathrm{n}=6) .{ }^{\#} P<0.05,{ }^{\#} P<0.01$ compared with control group; ${ }^{*} P<0.05,{ }^{* *} P<0.01$ compared with model group. $a$-SMA alpha smooth muscle actin, TGF- $\beta 1$ transforming growth factor-beta 
metabolic analysis and subsequently screening for metabolic differences.

\section{Quality control}

QC can determine whether the systematic error of the whole experiment is within the controllable range. As shown in Fig. 4, the cluster analysis of QC samples relative to the experimental samples showed that the QC samples were closely clustered, especially in positive ion mode. The above results prove that the method has good stability and repeatability.

\section{Multivariate statistical analysis}

Firstly, SIMCA-14.1 software was used to distinguish the control, model and $10.8 \mathrm{~g} / \mathrm{kg}$ of AR groups in the serum metabolic phenotypes and metabolites by PCA. The purpose of PCA was description, which is used to observe the separation between different groups of metabolites $[25,26]$. The results showed that a significant classification between the clustering of the control and model groups and the control and AR groups was observed in both the ESI+ and ESI- modes (Fig. 5). As shown in the Additional file 2: Figure S2, the corresponding loading scores plots from PCA could well summarize the influence of variables on the model. Subsequently, multivariate analysis was used to explore which metabolites contributed to these differences.

OPLS-DA, as a pattern recognition approach, was designed for predictions to identify differential metabolites that were significantly changed between control, model and $10.8 \mathrm{~g} / \mathrm{kg}$ of AR groups [25, 26]. The OPLSDA could only be used for screening differentially expressed metabolites between the two groups [27]. And this study aimed to explore the specific metabolites regulated by AR in rats with liver fibrosis. Therefore, we respectively identified differential metabolites from control vs model groups and model vs $10.8 \mathrm{~g} / \mathrm{kg}$ AR groups in OPLS-DA mode. Metabolites identified between the different groups are listed in Additional file 3: Tables S2 and S3. The same differential metabolites were chosen for subsequent analysis. In the OPLS-DA mode established from the serum data of the control and model groups, the variance of the response variable $\left(R^{2} Y\right)$ in the positive and negative modes were 1 and 1 , and the variance for modeling in cross-validations $\left(\mathrm{Q}^{2}\right)$ were 0.774 and 0.777 , respectively (Fig. 6a and Additional file 4: Figure S3A). For serum data of the model and AR groups in positive and negative modes, $R^{2} Y$ values were 0.999 and 0.999
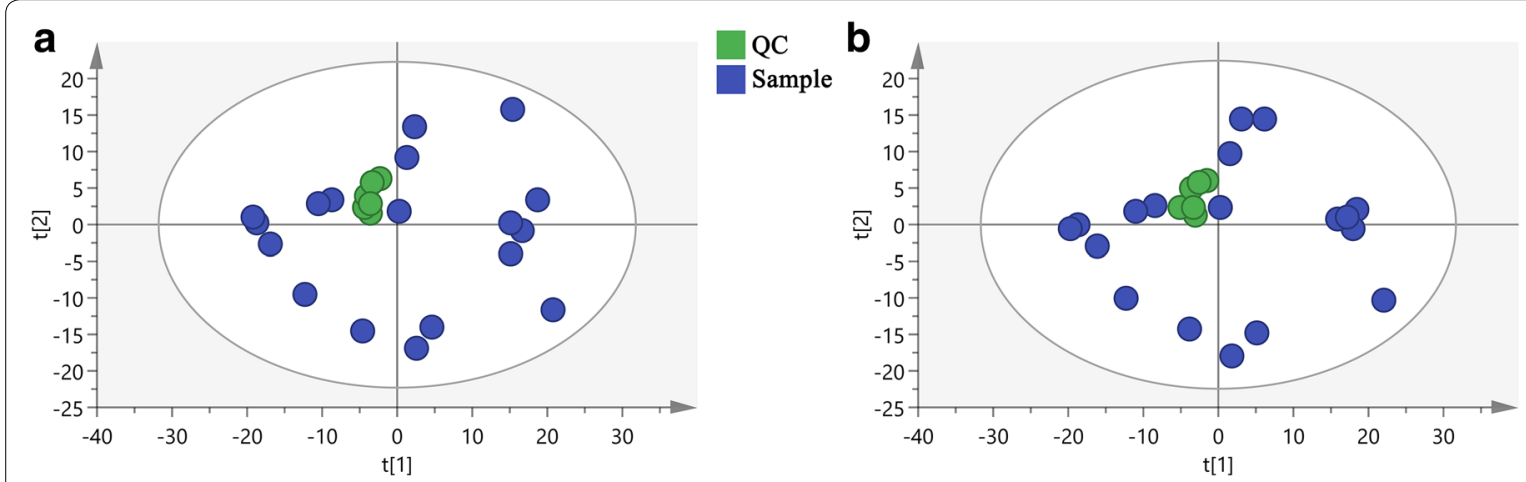

QC

Sample

Fig. 4 Principal component analysis (PCA) score plot of quality control (QC) samples. a ESI+ mode. b ESI- mode
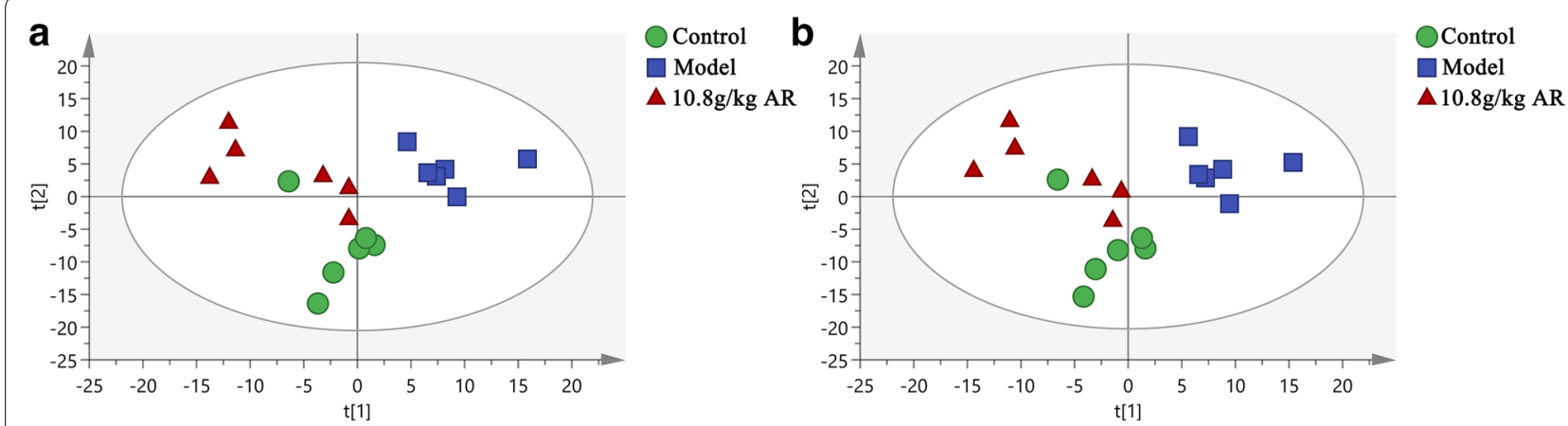

Fig. 5 Principal component analysis (PCA) score plot of normal, model and $10.8 \mathrm{~g} / \mathrm{kg}$ AR groups. a ESI+ mode. b ESI- mode 


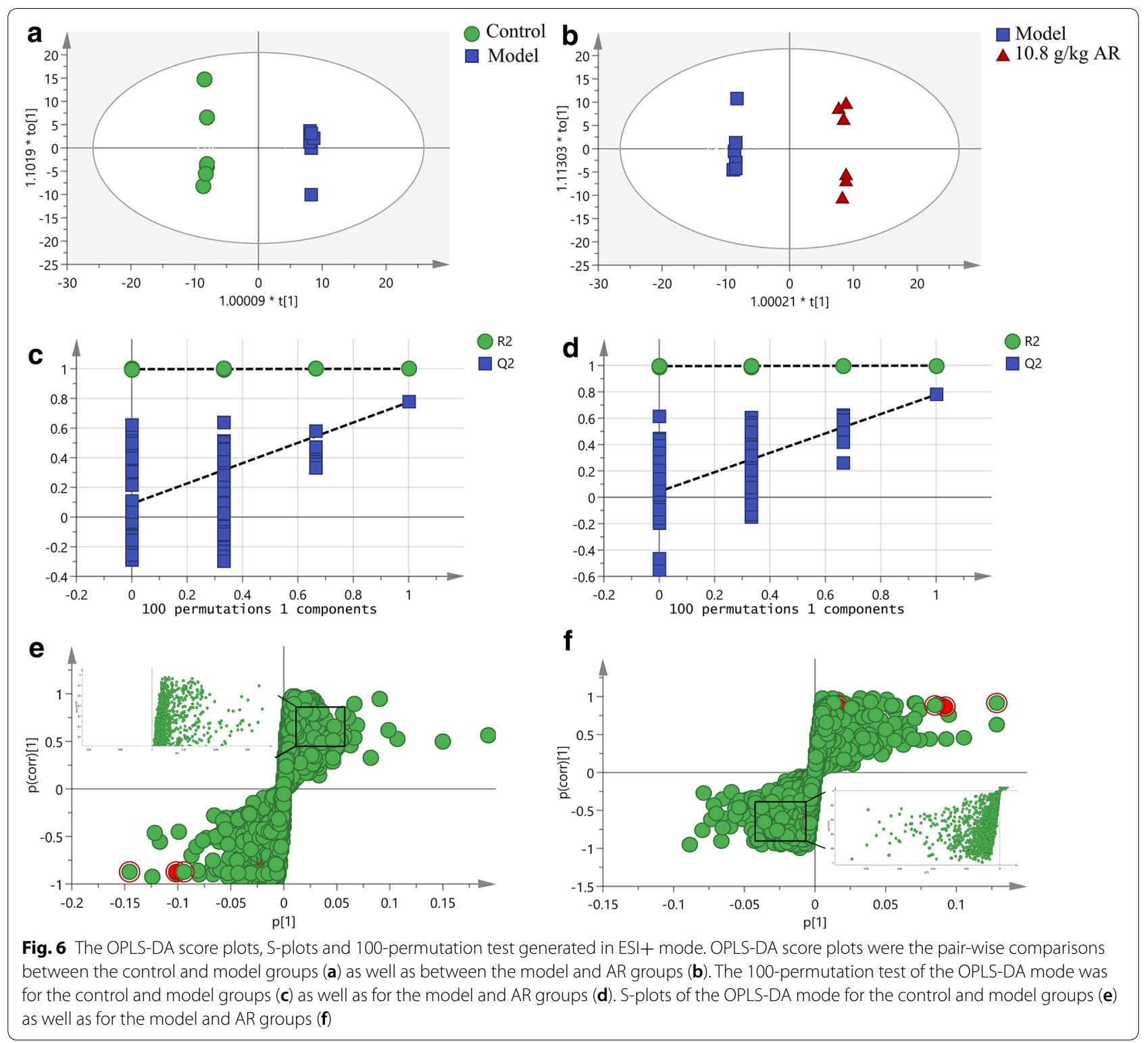

with $\mathrm{Q}^{2} 0.778$ and 0.778 , individually (Fig. $6 \mathrm{~b}$ and Additional file 4: S3B). The relevant parameter demonstrated that the modes had good explanatory and predictive ability. The permutation tests $(n=100)$ were performed to validate the predictive ability of modes (Fig. 6c, $\mathrm{d}$ and Additional file 4: S3C, D). The results showed that all $R^{2}$ and $\mathrm{Q}^{2}$ values were lower than that in the permutation tests, demonstrating the goodness of fit and better predictability of the OPLS-DA mode. The S-plot was used to study the inherent clustering variables. In the corresponding S-plot, variables whose VIP value were over 1 at average and $\mathrm{P}$ (corr) absolute values over 0.58 means can be considered as potential biomarkers (Fig. 6e, $\mathrm{f}$ and Additional file 4: S3E, F). In order to characterize differential metabolites more comprehensively, the potential differential metabolites obtained under ESI+ and ESI- modes were combined for subsequent analysis [28].

\section{Identification of potential metabolites in AR treatment}

During the potential metabolite identification, 595 potential differential metabolites were selected based on the principles that VIP $>1.0$ and $\mid \mathrm{P}$ (corr) $\mid \geq 0.58$ in the S-plots as candidates for ANOVA analysis. Candidate variables with significant differences were then screened using ANOVA analysis. Candidates with significant changes were identified as biomarkers based on the METLIN and Metaboanalyst databases. A total of 9 
metabolic differences were identified, 7 in ESI+ mode and 2 in ESI- mode, which were marked with red in the S-plots diagram (Fig. 6E, F and Additional file 4: S3E, F). As shown in Fig. 7, the nine potential biomarkers were sphingosine (C00319), 6-keto-prostaglandin F1a (C05961), LysoPC (O-18:0) (C04317), 3-dehydrosphinganine (C02934), 5,6-epoxy-8,11,14-eicosatrienoic acid (C14768), leukotriene C4 (C02166), taurochenodesoxycholic acid (C05465), LysoPC (18:1 (9Z)) (C04230), 2-acetyl-1-alkyl-sn-glycero-3-phosphocholine (C04598). The distribution patterns of 9 potential metabolites in the three groups were visually displayed by heatmap. And vertical cluster analysis was used to demonstrate the difference between model and control groups and the equivalent efficacy of AR (Fig. 8b). The corresponding formula and parameters including retention time, $\mathrm{m} / \mathrm{z}$ and differences between groups were listed in Table 2.

\section{Pathway analysis}

To explore the mechanism of AR on liver fibrosis, the metabolic pathways were constructed by importing the identified potential metabolites into MetaboAnalyst 4.0. It could be concluded that five pathways including arachidonic acid metabolism, ether lipid metabolism, sphingolipid metabolism, glycerophospholipid metabolism and primary bile acid biosynthesis were responsible for regulating $\mathrm{CCl}_{4}$-induced liver fibrosis (Table 3 ). As shown in Fig. 8a, the top three pathways were arachidonic acid metabolism, ether lipid metabolism and sphingolipid metabolism, which played a key role in reflecting changes. The impact-values of metabolites were 0.051, 0.07375 and 0.15499 , respectively. The MetScape and KEGG pathway analysis showed that the nine metabolic differences and five pathways were directly or indirectly related, as shown in Additional file 5: Figure S4. Based on the above results, we mapped the signaling networks associated with differentially expressed metabolic pathways (Fig. 8c).

\section{"Potential metabolite-target-component" interactive network and analysis}

To visually reveal the interaction among the potential metabolites, protein targets and components of AR regulation, a potential metabolite-target-component interaction network was constructed by collecting drug targets and targets associated with potential metabolites. As shown in Fig. 9a, 54 components interacted with 927 targets and 8 metabolites were involved in the potential metabolite-target-component interaction network. The next analysis showed 6 drug targets, including 2-acylglycerol $\mathrm{O}$-acyltransferase 2 (Q3SYC2), cytochrome P450 1A2 (P05177), choline-phosphate cytidylyltransferase A (P49585), cytochrome P450 3A4 (P08684), cytochrome
P450 1B1 (Q16678), and cytochrome P450 2A6 (P11509), directly regulate the 3 potential metabolites (Fig. 9b). The 6 drug targets were directly regulated by the 6 chemical components of AR, including daidzein, nicotinic acid, kaempferol, coumarin, palmitic acid and quercetin (Fig. 9b).

\section{Verification of network pharmacology}

RT-PCR and LC-MS/MS methods were used to verify the accuracy of network pharmacology prediction results. As shown in Fig. 10a, b, the expression of CYP1A2 and PCYT1A were significantly reduced in the model group compared to the control group $(P<0.01)$. And the levels of CYP1A2 and PCYT1A were significantly up-regulated after AR treatment in a dose-dependent manner $(P<0.01)$. Furthermore, the expression of the CYP1B1 in $\mathrm{CCl}_{4}$-induced hepatic fibrosis rats was significantly inhibited after AR treatment $(P<0.01$, Fig. 10c). In Fig. $10 \mathrm{~d}$, e, the mass spectrometry data of AR aqueous extract and reference substance showed that quercetin and nicotinic acid existed in AR aqueous extract, and the related parameters were shown in Table 4.

\section{Discussion}

Liver fibrosis is a chronic progressive liver disease with complex pathological mechanisms and there are no safe and effective treatment tools $[1,4]$. The mean of combining metabolomics with network pharmacology to dig data extensively can effectively overcome these problems [24, 29]. Metabolomics analyzes and detects the levels of small molecules in body fluids to determine which compounds have significant abnormalities and to reveal the mechanisms of disease development [30]. Network pharmacology reveals the molecular mechanism of drug therapy by analyzing the interactions between chemical components and disease-related macromolecule targets [23]. Therefore, in this study, UPLC-Q-TOF/MS-method based rat serum metabolomics combined with network pharmacology was firstly used to clarify the protective effect of AR on liver fibrosis, which also provided an indepth understanding of the mechanism of AR on liver fibrosis.

Serum biochemical indicators showed that AR significantly reduced serum ALT, AST and AKP levels, restored liver function and effectively alleviated liver injury. HE staining and Masson staining also confirmed that AR could effectively reduce liver damage and collagen deposition in liver tissue, as well as improved liver fibrosis. In addition, this study used a variety of methods to detect the expression of two specific makers of liver fibrosis, including $\alpha$-SMA (a specific marker of hepatic stellate cell activation) and TGF- $\beta 1$ (an important factor of hepatic fibrosis induced by $\mathrm{CCl}_{4}$ ) [31]. The results confirmed that 

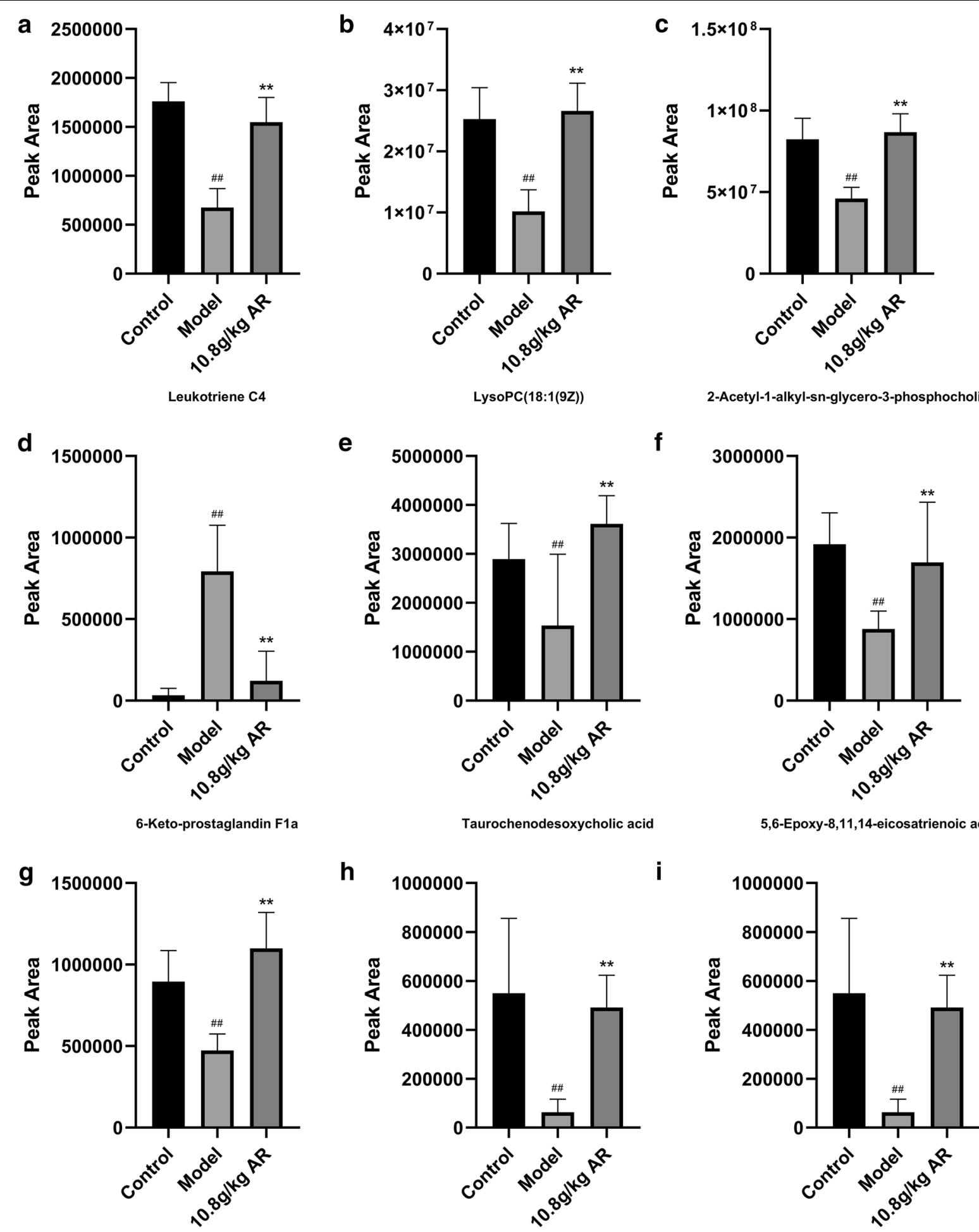

h
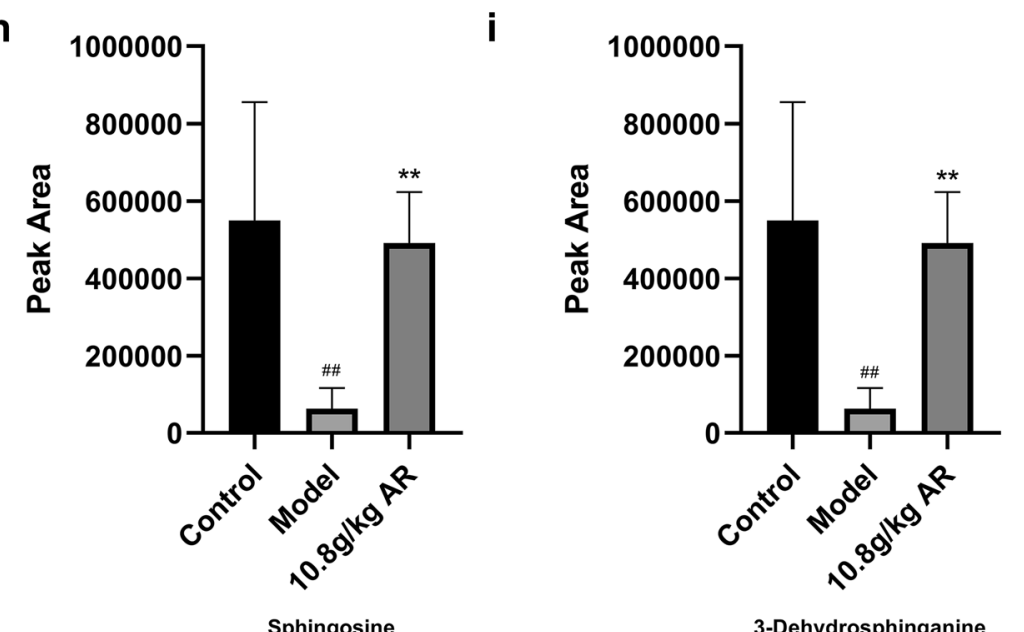

Fig. 7 Potential metabolites changes in CCl -induced liver injury treated by AR. a Leukotriene C4; b LysoPC(18:1 (9Z)); c 2-Acetyl-1-alkyl-sn-gly cero-3-phosphocholine; d 6-Keto-prostaglandin F1a; e Taurochenodesoxycholic acid; f 5,6-Epoxy-8,1 1,14-eicosatrienoic acid; g LysoPC(O-18:0);

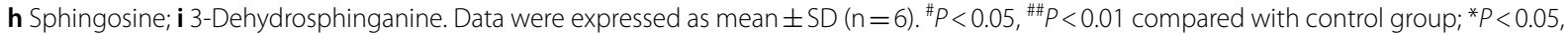
${ }^{* *} P<0.01$ compared with model group 


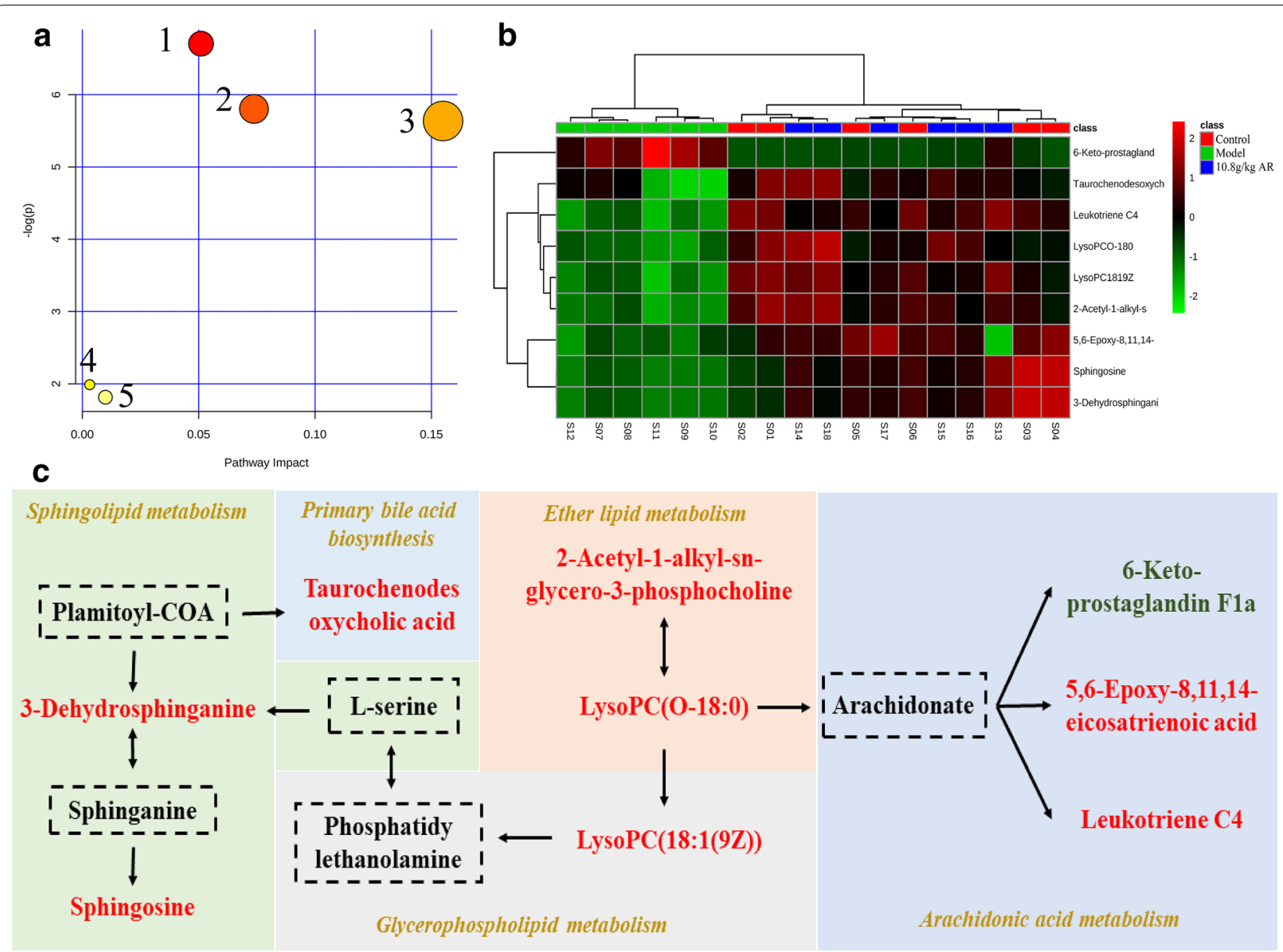

Fig. 8 Potential metabolomic pathway in $\mathrm{CCl}_{4}$-induced liver injury treated by AR. a Metabolomic pathway construction of the metabolic pathways involved in the effects of AR on liver fibrosis. b The heatmap of 9 potential metabolites. $\mathbf{c}$ Signaling networks associated with the differentially expressed metabolic pathways. 1: Arachidonic acid metabolism. 2: Ether lipid metabolism. 3: Sphingolipid metabolism. 4: Glycerophospholipid metabolism. 5: Primary bile acid biosynthesis

Table 2 Identified metabolites of the serum from different groups

\begin{tabular}{|c|c|c|c|c|c|c|c|c|}
\hline \multirow[t]{2}{*}{ No. } & \multirow[t]{2}{*}{ R.T. (min) } & \multirow[t]{2}{*}{ Mass $(\mathrm{m} / \mathrm{z})$} & \multirow[t]{2}{*}{ Metabolites } & \multirow[t]{2}{*}{ Formula } & \multirow[t]{2}{*}{ KEGG } & \multicolumn{2}{|l|}{ Change trend } & \multirow[t]{2}{*}{ Pathway } \\
\hline & & & & & & Control/model & Model/AR & \\
\hline 1 & 14.174 & 624.281 & Leukotriene C4 & $\mathrm{C}_{30} \mathrm{H}_{47} \mathrm{~N}_{3} \mathrm{O}_{9} \mathrm{~S}$ & C02166 & Down & Up & Arachidonic acid metabolism \\
\hline 2 & 14.224 & 544.339 & LysoPC(18:1 (9Z)) & $\mathrm{C}_{26} \mathrm{H}_{52} \mathrm{NO}_{7} \mathrm{P}$ & C04230 & Down & Up & $\begin{array}{l}\text { Glycerophospholipid } \\
\text { metabolism }\end{array}$ \\
\hline 3 & 16.987 & 524.371 & $\begin{array}{l}\text { 2-Acetyl-1-alkyl-sn-glycero- } \\
\text { 3-phosphocholine }\end{array}$ & $\mathrm{C}_{26} \mathrm{H}_{54} \mathrm{NO}_{7} \mathrm{P}$ & C04598 & Down & Up & Ether lipid metabolism \\
\hline 4 & 10.064 & 371.235 & 6-Keto-prostaglandin F1a & $\mathrm{C}_{20} \mathrm{H}_{34} \mathrm{O}_{6}$ & C05961 & Up & Down & Arachidonic acid metabolism \\
\hline 5 & 14.609 & 498.335 & Taurochenodesoxycholic acid & $\mathrm{C}_{26} \mathrm{H}_{45} \mathrm{NO}_{6} \mathrm{~S}$ & C05465 & Down & Up & Primary bile acid biosynthesis \\
\hline 6 & 16.363 & 343.224 & $\begin{array}{l}\text { 5,6-Epoxy-8,11,14-eicosatrien- } \\
\text { oic acid }\end{array}$ & $\mathrm{C}_{20} \mathrm{H}_{32} \mathrm{O}_{3}$ & C14768 & Down & Up & Arachidonic acid metabolism \\
\hline 7 & 16.030 & 532.340 & LysoPC(O-18:0) & $\mathrm{C}_{26} \mathrm{H}_{56} \mathrm{NO}_{6} \mathrm{P}$ & C04317 & Down & Up & Ether lipid metabolism \\
\hline 8 & 18.113 & 322.269 & Sphingosine & $\mathrm{C}_{18} \mathrm{H}_{37} \mathrm{NO}_{2}$ & C00319 & Down & Up & Sphingolipid metabolism \\
\hline 9 & 18.150 & 322.270 & 3-Dehydrosphinganine & $\mathrm{C}_{18} \mathrm{H}_{37} \mathrm{NO}_{2}$ & C02934 & Down & Up & Sphingolipid metabolism \\
\hline
\end{tabular}


Table 3 Results of integrating enrichment analysis of biomarkers with MetaboAnalyst 4.0

\begin{tabular}{|c|c|c|c|c|c|}
\hline No. & Pathway name & Match status & $P$ & $-\log (p)$ & Impact \\
\hline 1 & Arachidonic acid metabolism & $3 / 62$ & 0.0012245 & 6.7052 & 0.051 \\
\hline 2 & Ether lipid metabolism & $2 / 23$ & 0.0030196 & 5.8026 & 0.07375 \\
\hline 3 & Sphingolipid metabolism & $2 / 25$ & 0.0035667 & 5.6361 & 0.15499 \\
\hline 4 & Glycerophospholipid metabolism & $1 / 39$ & 0.13694 & 1.9882 & 0.00317 \\
\hline 5 & Primary bile acid biosynthesis & $1 / 47$ & 0.16287 & 1.8148 & 0.00992 \\
\hline
\end{tabular}
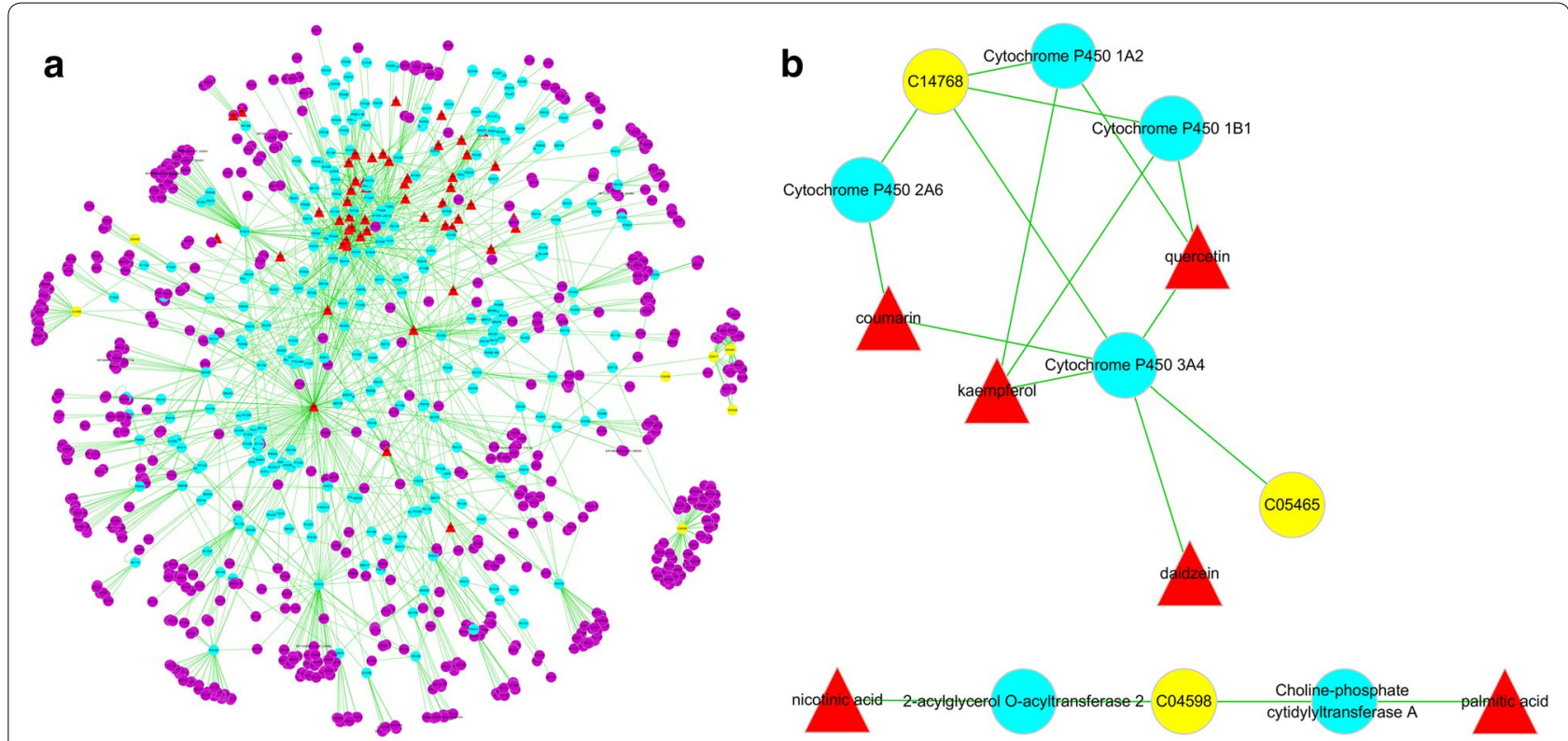

Fig. 9 The "potential metabolite-target-component" interaction network with (a) all target information and (b) key target information participating in the treatment of liver fibrosis by AR. The red triangles represent active chemical constituents of AR. The blue dots represent the protein targets of drugs. The yellow dots represent potential metabolites. The purple dots represent the targets associated with potential metabolites

AR could effectively reduce the expression of the two markers. The above results confirmed that AR exhibited significant anti-hepatic fibrosis effect, which was consistent with clinical observation.

Subsequently, the metabolomic profiles of AR in the treatment of liver fibrosis were described. AR protected liver fibrosis by reversing potential metabolites to normal levels. Biomarkers can be an effective method for disease diagnosis, treatment and prognosis [32]. Metabolism biomarkers were identified in the OPLS-DA mode. There were significant differences of biomarkers between the control group and the model group, which indicated that the serum metabolomics profile was significantly affected by $\mathrm{CCl}_{4}$. At the same time, significant differences were observed between the AR group and the model group, which indicated the regulatory role of AR. Finally, nine differential metabolites were screened to reveal the regulatory mechanism of $\mathrm{AR}$ in ameliorating liver fibrosis. These metabolites can interact in different ways. The results suggest that the development and progression of liver fibrosis were caused by the changes in many physiological and pathologically related molecules, and most of the changes in the body are interrelated. AR modulated these nine biomarkers to normalize their expression levels, indicating that AR can treat liver fibrosis through multiple pathways and multiple targets.

Those metabolites involved five main metabolic pathways including arachidonic acid metabolism, ether lipid metabolism, sphingolipid metabolism, glycerophospholipid metabolism and primary bile acid biosynthesis. The decomposition and metabolism of arachidonic acid plays a major role in triggering and eliminating inflammation [33]. Sustained inflammatory response is the key to the progress of liver fibrosis. Studies have shown that AR can improve liver fibrosis by regulating arachidonic acid metabolism. Ether lipids are a unique class of glycerophospholipids, which account for about $20 \%$ of mammalian total phospholipids and are present in 

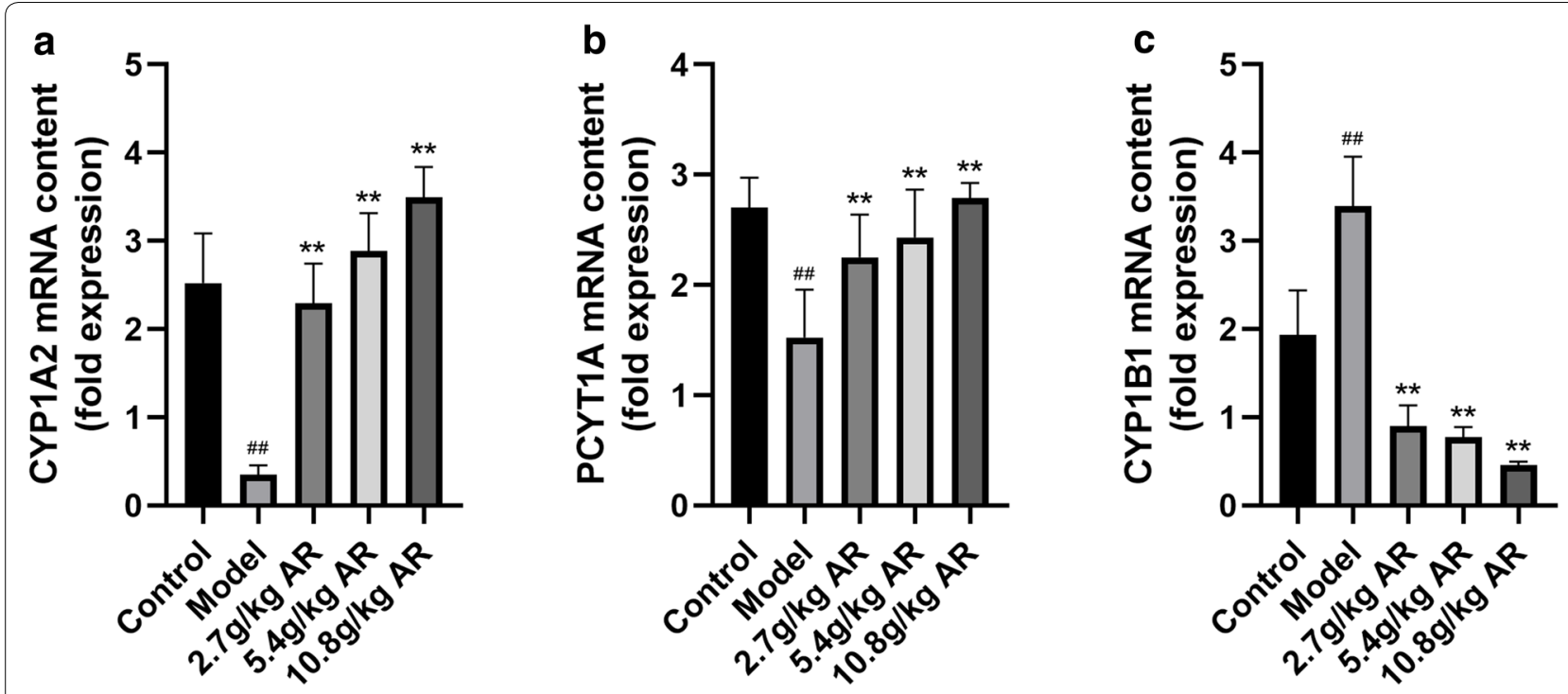

d

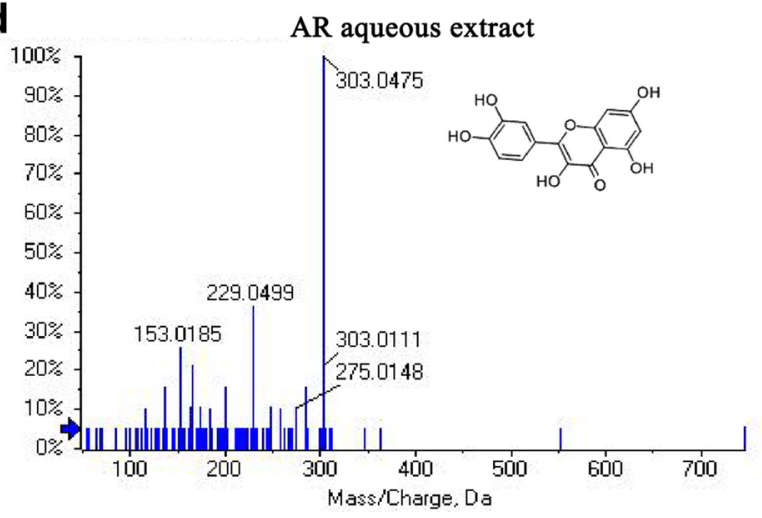

e

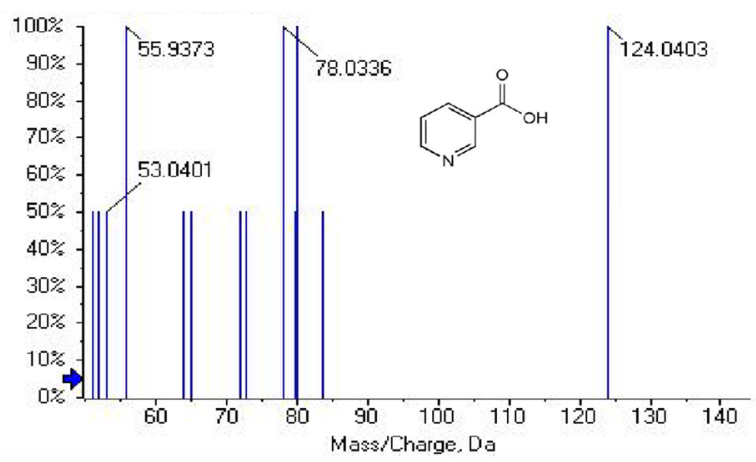

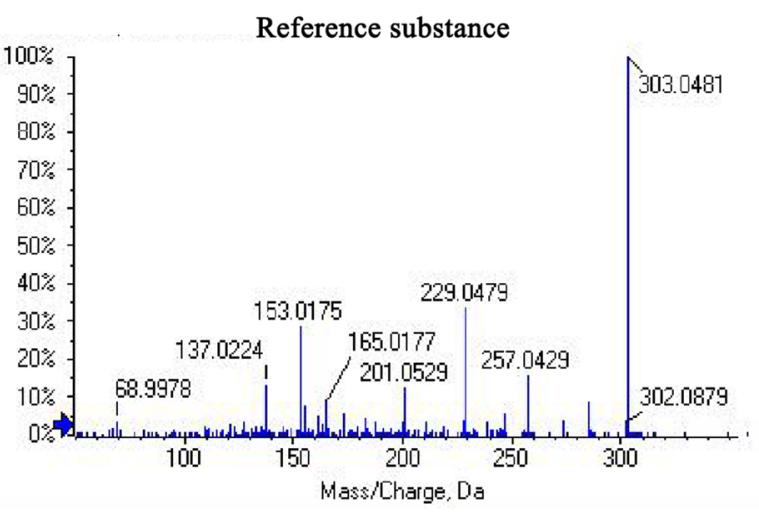

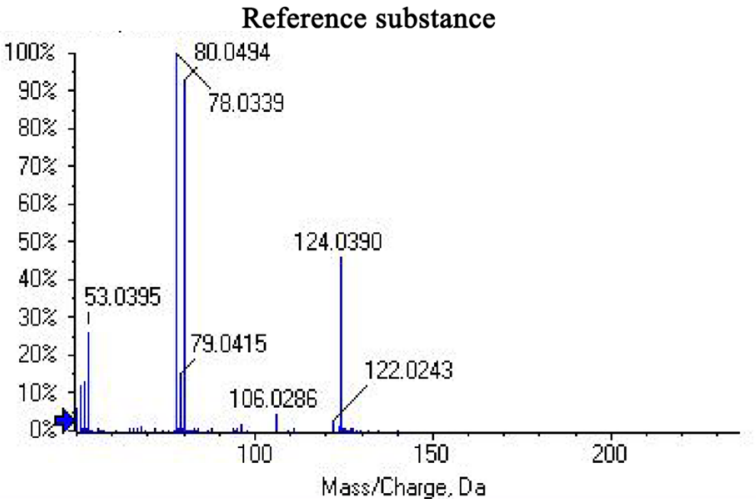

Fig. 10 The verification results of network pharmacology. a The expression of CYP1A2. b The expression of PCYT1A. c The expression of CYP1B1. d The mass spectrum of quercetin. e The mass spectrum of nicotinic acid. Data were expressed as mean $\pm S D(n=6) .{ }^{\#} P<0.05,{ }^{\# \#} P<0.01$ compared with control group; ${ }^{*} P<0.05,{ }^{* *} P<0.01$ compared with model group

Table 4 The parameters of the active compounds in the AR extract

\begin{tabular}{llllll}
\hline No. & Compound & Formula & Extraction mass & Found mass & RT (min) \\
\hline 1 & Quercetin & $\mathrm{C}_{15} \mathrm{H}_{10} \mathrm{O}_{7}$ & 303.04993 & 303.04926 & -2.2 \\
2 & Nicotinic acid & $\mathrm{C}_{6} \mathrm{H}_{5} \mathrm{NO}_{2}$ & 124.03930 & 124.03934 & 0.3 \\
\hline
\end{tabular}


small amounts in the liver [34]. Studies have shown that ether lipid metabolism was associated with liver damage caused by steatosis. Acetaldehyde phosphatide may prevent steatosis and non-alcoholic steatohepatitis through anti-oxidative stress [35, 36]. Sphingolipid metabolites played a key role in inflammatory signal. Studies have confirmed that sphingolipid metabolism was associated with hepatic lipid degeneration and inflammation [37]. Metabolic results suggested that AR played a protective role in liver by regulating sphingolipid metabolism. In addition, studies have proved that both glycerophospholipid metabolism and primary bile acid biosynthesis were closely related to liver injury. Glycerophospholipid metabolism played a key role in liver injury [38]. Bile acid is synthesized by cholesterol in the liver, and its synthesis and secretion can reflect liver function. In addition, bile acid can destroy cell membranes through its decontamination and promote oxidative stress through reactive oxygen species production, leading to hepatocyte and non-parenchymal cell damage [39].

In order to better understand the mechanism of AR in the treatment of liver fibrosis and the correlation between the AR chemical constituents and metabolites, a metabolite-target-component interaction network in combination with network pharmacology was further constructed. The results showed that six chemical components directly acted on a variety of targets, including CYP1A2, PCYT1A, CYP3A4, CYP2A6, MOGAT2 and CYP1B1, and were directly related to a variety of metabolites. CYP1A2 is a member of the CYP450s family and plays a crucial role in liver metabolism [40]. It has been found that the activity of CYP1A2 decreases with the degree of fibrosis in non-tumorous liver tissues [41]. CYP1B1 is also a member of CYP450s, which exists in hepatic endothelial cells and activated stellate cells and is involved in the metabolism of many important physiological compounds [42]. Studies have shown that hepatic steatosis and tumorigenesis could be reduced by inhibiting CYP1B1 [43]. Metabolomics combined with network pharmacological analysis showed that CYP1A2 and CYP1B1 directly regulate 5,6-Epoxy-8,11,14-eicosatrienoic acid, which participates in arachidonic acid metabolism. These results demonstrated that AR might ameliorate liver fibrosis by regulating the liver CYP450s in vivo for the potential therapeutic control of arachidonic acid metabolism. PCYT1A is the rate-limiting enzyme in the synthesis of phosphatidylcholine (PC), and $\mathrm{PC}$ is an essential component in all cell membranes. Previous studies have reported that $\mathrm{pcyt}^{-1-}$ mice exhibited severe fatty liver, dyslipidemia and other phenotypic characteristics [44]. MOGAT2 plays an important role in the process of fat absorption and metabolism. It is reported that mogat $2^{-1-}$ mice are protected from hepatic steatosis induced by high-fat diet $[45,46]$. Network pharmacological analysis showed that PCYT1A and MOGAT2 act directly on 2-Acetyl-1-alkyl-snglycero-3-phosphocholine, which participates in ether lipid metabolism. And these results indicated that AR could modulate ether lipid metabolism to ameliorate $\mathrm{CCl}_{4}$-induced hepatic steatosis and fibrosis by regulating PCYT1A and MOGAT2.

The results of RT-PCR in liver tissue of rats with liver fibrosis showed that the expression of CYP1A2 and PCYT1A was up-regulated and the expression of CYP1B1 was down-regulated in AR treatment groups, which was consistent with the previously reported results. The mRNA expression of CYP3A4, CYP2A6 and MOGAT2 was not detected, probably because the results of network pharmacology came from big data, and not all results could be verified. It might also be due to improper detection methods. Mass spectrometry data indicated that AR extract contained quercetin and nicotinic acid, which might play an important role in the treatment of liver fibrosis. The other four components were not detected, which might be due to the change of compounds during AR extraction. It might also be that the content of those components in AR extract was too low to be detected. These results not only indicated that AR could ameliorate liver fibrosis by regulating the expression of CYP1A2, PCYT1A and CYP1B1, but also confirmed that the predicted results of network pharmacology were credible.

Taken together, our study found that AR could be involved in the regulation of arachidonic acid metabolism and ether lipid metabolism by regulating the expression of CYP1A2, PCYT1A and CYP1B1, thereby effectively improving liver fibrosis. This study confirmed the advantages of AR in the treatment of liver fibrosis with multiple targets and multiple pathways. In addition, it has been reported that Asiatic acid can ameliorate $\mathrm{CCl}_{4}$-induced liver fibrosis in rats by regulating PI3K/ AKT/mTOR, Bcl-2/Bax, Nrf2/ARE, NF-kB/IkB $\alpha$ and JAK1/STAT3 signaling pathways $[47,48]$. Salvia miltiorrhiza could inhibit liver fibrosis by regulating TGF- $\beta$ / Smad, Nrf2/HO-1 and NF-кB/ІкB $\alpha$ signaling pathways $[49,50]$. These results indicate that multi-channel and multi-target are the advantages of TCM, which has great potential in the treatment of liver fibrosis and deserves further exploration.

\section{Conclusions}

This study confirmed that AR had a therapeutic effect on $\mathrm{CCl}_{4}$-induced liver fibrosis, possibly by regulating arachidonic acid metabolism and ether lipid metabolism, which may provide new insights into the mechanism of $\mathrm{AR}$ in the treatment of liver fibrosis. In addition, this 
study also confirmed that TCM has potential advantages in the treatment of liver fibrosis, which is worth further exploring.

\section{Supplementary information}

Supplementary information accompanies this paper at https://doi. org/10.1186/s13020-019-0251-z.

Additional file 1: Figure S1. Q -TOF LC/MS total ion chromatogram in ESI+. Table S1. The major chemical compounds of AR extract.

Additional file 2: Figure S2. The corresponding loading scores plots from PCA.

Additional file 3: Table S2. Differential identified metabolites for discrimination among control and model groups. Table S3. Differential identified metabolites for discrimination among model and $10.8 \mathrm{~g} / \mathrm{kg}$ AR groups.

Additional file 4: Figure S3. The OPLS-DA score plots, S-plots and 100-permutation test generated in ESI- mode.

Additional file 5: Figure S5. The interrelation between metabolic differences.

\section{Abbreviations}

AR: Astragali Radix; ALT: alanine transaminase; AST: aspartate transaminase; AKP: alkaline phosphatase; $a-S M A$ : alpha smooth muscle actin; TGF- $\beta 1$ : transforming growth factor-beta 1; PBS: phosphate buffered saline; QC: quality control; UPLC-Q-TOF/MS: ultraperformance liquid chromatography with quadrupole time-of flight mass spectrometry; PCA: principle component analysis; OPLS-DA: orthogonal projection to latent structures discriminate analysis; ESI: electrospray ionization; KEGG: Kyoto Encyclopedia of Genes of Genomes; TCMSP: traditional Chinese medicine systems pharmacology; MOGAT2: 2-acylglycerol O-acyltransferase 2; CYP4501A2: cytochrome P4501A2; PCYTIA: choline-phosphate cytidylyltransferase A; CYP3A4: cytochrome P4503A4; CYP4501B1: cytochrome P4501B1; CYP2A6: cytochrome P450 2A6; RT-PCR: real time polymerase chain reaction; $\mathrm{CCl}_{4}$ : carbon tetrachloride; $\mathrm{H} \& \mathrm{E}$ : hematoxylin-eosin; HMDB: Human Metabolome Database; PPI: protein interaction; TCM: traditional Chinese medicine.

\section{Acknowledgements}

Not applicable.

\section{Author's contributions}

DW, RL, SW, and SG conducted experiments and wrote manuscript. The data was analyzed by $Z X, H L$ and RW. HL and HC revised the paper. JW and $Y Z$ designed the study and guided the experiment. All authors read and approved the final manuscript.

\section{Funding}

This work was funded by the project of Capital's Funds for Health Improvement and Research (No. 2018-2-5031).

\section{Availability of data and materials}

The data used to support the findings of this study are available from the corresponding author upon request.

\section{Ethics approval and consent to participate}

All experimental protocols described in the present study were approved by the Ethical Committee of Fifth Medical Center of PLA General Hospital of China. All procedures for the animal study were conducted in accordance with ARRIVE guidelines, and every effort was made to alleviate the suffering of the animals.

\section{Consent for publication}

Not applicable.

\section{Competing interests}

The authors declared that they have no competing interests.

\section{Author details}

${ }_{1}^{1}$ Provincial and State Key Laboratory Breeding Base of System Research, Development and Utilization of Chinese Herbal Medicine Resources, College of Pharmacy, Chengdu University of Traditional Chinese Medicine, Chengdu 611137, China. ${ }^{2}$ Department of Pharmacy, The Fifth Medical Center of PLA General Hospital, Beijing 100039, China. ${ }^{3}$ Research Center for Clinical and Translational Medicine, The Fifth Medical Center of PLA General Hospital, Beijing 100039, China. ${ }^{4}$ Integrative Medical Center, The Fifth Medical Center of PLA General Hospital, Beijing 100039, China. ${ }^{5}$ Department of Traditional Chinese Medicine, The Fifth Medical Center of PLA General Hospital, Beijing 100039, China.

Received: 25 June 2019 Accepted: 14 August 2019

Published online: 28 August 2019

\section{References}

1. Xie H, Su D, Zhang J, Ji D, Mao J, Hao M, et al. Raw and vinegar processed Curcuma wenyujin regulates hepatic fibrosis via bloking TGF-beta/Smad signaling pathways and up-regulation of MMP-2/TIMP-1 ratio. J Ethnopharmacol. 2019. https://doi.org/10.1016/j.jep.2019.01.045

2. Lei Y, Wang QL, Shen L, Tao YY, Liu CH. MicroRNA-101 suppresses liver fibrosis by downregulating PI3K/Akt/mTOR signaling pathway. Clin Res Hepatol Gastroenterol. 2019;19:30041-5. https://doi.org/10.1016/j.clinr e.2019.02.003

3. Schuppan D, Ashfaq-Khan M, Yang AT, Kim YO. Liver fibrosis: direct antifibrotic agents and targeted therapies. Matrix Biol. 2018;68-69:435-51.

4. Yoon YJ, Friedman SL, Lee YA. Antifibrotic therapies: where are we now? Semin Liver Dis. 2016;36(1):87-98.

5. Li AP, Li ZY, Sun HF, Li K, Qin XM, Du GH. Comparison of two different Astragali Radix by a (1)H NMR-based metabolomic approach. J Proteome Res. 2015;14(5):2005-16.

6. Liu L, Leng J, Yang X, Liao L, Cen Y, Xiao A, et al. Rapid screening and identification of BSA bound ligands from radix astragali using BSA immobilized magnetic nanoparticles coupled with HPLC-MS. Molecules. 2016;21(11):1471.

7. Gong AGW, Duan R, Wang HY, Kong XP, Dong TTX, Tsim KWK, et al. Evaluation of the pharmaceutical properties and value of Astragali Radix. Medicines. 2018;5(2):46.

8. Zhou Y, Tong X, Ren S, Wang X, Chen J, Mu Y, et al. Synergistic anti-liver fibrosis actions of total astragalus saponins and glycyrrhizic acid via TGF-beta1/Smads signaling pathway modulation. J Ethnopharmacol. 2016:190:83-90.

9. Huang W, Li L, Tian X, Yan J, Yang X, Wang X, et al. Astragalus and Paeoniae radix rubra extract inhibits liver fibrosis by modulating the transforming growth factorbeta/Smad pathway in rats. Mol Med Rep. 2015;11(2):805-14.

10. Simeonova R, Bratkov VM, Kondeva-Burdina M, Vitcheva V, Manov V, Krasteva I. Experimental liver protection of n-butanolic extract of Astragalus monspessulanus L. on carbon tetrachloride model of toxicity in rat. Redox Rep. 2015;20(4):145-53.

11. Vitcheva V, Simeonova R, Krasteva I, Nikolov S, Mitcheva M. Protective effects of a purified saponin mixture from Astragalus corniculatus Bieb., in vivo hepatotoxicity models. Phytother Res. 2013;27(5):731-6.

12. Allam RM, Selim DA, Ghoneim Al, Radwan MM, Nofal SM, Khalifa AE, et al. Hepatoprotective effects of Astragalus kahiricus root extract against ethanol-induced liver apoptosis in rats. Chin J Nat Med. 2013;11(4):354-61.

13. Lee JS, Cho JH, Lee DS, Son CG. genotoxicity evaluation of an ethanol extract mixture of Astragali Radix and salviae miltiorrhizae radix. Evid Based Complement Alternat Med. 2018;2018:5684805.

14. Yu SY, Ouyang HT, Yang JY, Huang XL, Yang T, Duan JP, et al. Subchronic toxicity studies of Radix Astragali extract in rats and dogs. J Ethnopharmacol. 2007;110(2):352-5.

15. Wang $X$, Zhang A, Sun H. Power of metabolomics in diagnosis and biomarker discovery of hepatocellular carcinoma. Hepatology. 2013;57(5):2072-7.

16. Zhang $Y$, Zhang M, Li H, Zhao H, Wang F, He Q, et al. Serum metabonomics study of the hepatoprotective effect of amarogentin on CCl4-induced liver fibrosis in mice by GC-TOF-MS analysis. J Pharm Biomed Anal. 2018;149:120-7. 
17. Su B, Luo P, Yang Z, Yu P, Li Z, Yin P, et al. A novel analysis method for biomarker identification based on horizontal relationship: identifying potential biomarkers from large-scale hepatocellular carcinoma metabolomics data. Anal Bioanal Chem. 2019. https://doi.org/10.1007/s0021 6-019-02011-w.

18. Yan Y, Du C, Li Z, Zhang M, Li J, Jia J, et al. Comparing the antidiabetic effects and chemical profiles of raw and fermented Chinese Ge-Gen-QinLian decoction by integrating untargeted metabolomics and targeted analysis. Chin Med. 2018;13:54.

19. Zhang Y, Li W, Zou L, Gong Y, Zhang P, Xing S, et al. Metabonomic study of the protective effect of Fukeqianjin formula on multi-pathogen induced pelvic inflammatory disease in rats. Chin Med. 2018;13:61.

20. Li A-P, Li Z-Y, Qu T-L, Qin X-M, Du G-H. Nuclear magnetic resonance based metabolomic differentiation of different Astragali Radix. Chin J Nat Med. 2017;15(5):363-74.

21. Lyu M, Zhou Z, Wang X, Lv H, Wang M, Pan G, et al. Network pharmacology-guided development of a novel integrative regimen to prevent acute graft-vs.-host disease. Front Pharmacol. 2018;9:1440.

22. Zhu B, Zhang W, Lu Y, Hu S, Gao R, Sun Z, et al. Network pharmacologybased identification of protective mechanism of Panax notoginseng saponins on aspirin induced gastrointestinal injury. Biomed Pharmacother. 2018;105:159-66.

23. Zhang R, Zhu X, Bai H, Ning K. Network pharmacology databases for Traditional Chinese Medicine: review and assessment. Front Pharmacol. 2019;10:123.

24. Wei S, Qian L, Niu M, Liu H, Yang Y, Wang Y, et al. The modulatory properties of Li-Ru-Kang treatment on hyperplasia of mammary glands using an integrated approach. Front Pharmacol. 2018;9:651.

25. Worley B, Powers R. PCA as a practical indicator of OPLS-DA model reliability. Curr Metab. 2016;4(2):97-103.

26. Worley B, Powers R. Multivariate analysis in metabolomics. Curr Metab. 2013;1(1):92-107.

27. Blasco H, Blaszczynski J, Billaut JC, Nadal-Desbarats L, Pradat PF, Devos D, et al. Comparative analysis of targeted metabolomics: dominance-based rough set approach versus orthogonal partial least square-discriminant analysis. J Biomed Inform. 2015;53:291-9.

28. Creydt M, Fischer M. Plant metabolomics: maximizing metabolome coverage by optimizing mobile phase additives for nontargeted mass spectrometry in positive and negative electrospray ionization mode. Anal Chem. 2017;89(19):10474-86.

29. Wang X, Zhang C, Zheng M, Gao F, Zhang J, Liu F. Metabolomics analysis of L-arginine induced gastrointestinal motility disorder in rats using UPLCMS after magnolol treatment. Front Pharmacol. 2019;10:183.

30. Kim DJ, Chung H, Ji SC, Lee S, Yu KS, Jang IJ, et al. Ursodeoxycholic acid exerts hepatoprotective effects by regulating amino acid, flavonoid, and fatty acid metabolic pathways. Metabolomics. 2019;15(3):30.

31. Yu J, He JQ, Chen DY, Pan QL, Yang JF, Cao HC, et al. Dynamic changes of key metabolites during liver fibrosis in rats. World J Gastroenterol. 2019:25(8):941-54.

32. Jiang W, Si L, Li P, Bai B, Qu J, Hou B, et al. Serum metabonomics study on antidiabetic effects of fenugreek flavonoids in streptozotocin-induced rats. J Chromatogr B. 2018;1092:466-72.

33. Gai Z, Visentin M, Gui T, Zhao L, Thasler WE, Hausler S, et al. Effects of farnesoid $X$ receptor activation on arachidonic acid metabolism, NF-kB signaling, and hepatic inflammation. Mol Pharmacol. 2018;94(2):802-11.

34. Dean JM, Lodhi IJ. Structural and functional roles of ether lipids. Protein Cell. 2018;9(2):196-206.

35. Barr J, Caballeria J, Martinez-Arranz I, Dominguez-Diez A, Alonso C, Muntane J, et al. Obesity-dependent metabolic signatures associated with nonalcoholic fatty liver disease progression. J Proteome Res. 2012;11(4):2521-32
36. Jang JE, Park HS, Yoo HJ, Baek IJ, Yoon JE, Ko MS, et al. Protective role of endogenous plasmalogens against hepatic steatosis and steatohepatitis in mice. Hepatology. 2017:66(2):416-31.

37. Norris GH, Blesso CN. Dietary and endogenous sphingolipid metabolism in chronic inflammation. Nutrients. 2017:9(11):1180.

38. Zhou C, Jia HM, Liu YT, Yu M, Chang X, Ba YM, et al. Metabolism of glycerophospholipid, bile acid and retinol is correlated with the early outcomes of autoimmune hepatitis. Mol BioSyst. 2016;12(5):1574-85.

39. Fader KA, Nault $R$, Zhang $C$, Kumagai $K$, Harkema JR, Zacharewski TR. 2,3,7,8-Tetrachlorodibenzo-p-dioxin (TCDD)-elicited effects on bile acid homeostasis: alterations in biosynthesis, enterohepatic circulation, and microbial metabolism. Sci Rep. 2017;7(1):5921.

40. Zhou J, Wen Q, Li SF, Zhang YF, Gao N, Tian X, et al. Significant change of cytochrome P450s activities in patients with hepatocellular carcinoma. Oncotarget. 2016;7(31):50612-23.

41. Wuensch T, Heucke N, Wizenty J, Quint J, Sinn B, Arsenic R, et al. Hepatic CYP1A2 activity in liver tumors and the implications for preoperative volume-function analysis. Am J Physiol Gastrointest Liver Physiol. 2019;316(5):G608-14

42. Larsen MC, Bushkofsky JR, Gorman T, Adhami V, Mukhtar H, Wang S, et al. Cytochrome P450 1B1: an unexpected modulator of liver fatty acid homeostasis. Arch Biochem Biophys. 2015;571:21-39.

43. Li F, Zhu W, Gonzalez FJ. Potential role of CYP1B1 in the development and treatment of metabolic diseases. Pharmacol Ther. 2017;178:18-30.

44. Payne F, Lim K, Girousse A, Brown RJ, Kory N, Robbins A, et al. Mutations disrupting the Kennedy phosphatidylcholine pathway in humans with congenital lipodystrophy and fatty liver disease. Proc Natl Acad Sci USA 2014;111(24):8901-6.

45. Yen CL, Cheong ML, Grueter C, Zhou P, Moriwaki J, Wong JS, et al. Deficiency of the intestinal enzyme acyl CoA:monoacylglycerol acyltransferase-2 protects mice from metabolic disorders induced by high-fat feeding. Nat Med. 2009;15(4):442-6.

46. Devasthale $P$, Cheng D. Monoacylglycerol acyltransferase 2 (MGAT2) inhibitors for the treatment of metabolic diseases and nonalcoholic steatohepatitis (NASH). J Med Chem. 2018;61(22):9879-88.

47. Fan J, Chen Q, Wei L, Zhou X, Wang R, Zhang H. Asiatic acid ameliorates CCl4-induced liver fibrosis in rats: involvement of Nrf2/ARE, NF-kappaB/ IkappaBalpha, and JAK1/STAT3 signaling pathways. Drug Des Devel Ther. 2018;12:3595-605.

48. Wei L, Chen Q, Guo A, Fan J, Wang R, Zhang H. Asiatic acid attenuates CCl4-induced liver fibrosis in rats by regulating the PI3K/AKT/mTOR and BCl-2/Bax signaling pathways. Int Immunopharmacol. 2018:60:1-8.

49. He S, Yang Y, Liu X, Huang W, Zhang X, Yang S, et al. Compound Astragalus and Salvia miltiorrhiza extract inhibits cell proliferation, invasion and collagen synthesis in keloid fibroblasts by mediating transforming growth factor-beta/Smad pathway. Br J Dermatol. 2012;166(3):564-74.

50. Wang R, Wang J, Song F, Li S, Yuan Y. Tanshinol ameliorates CCl4-induced liver fibrosis in rats through the regulation of Nrf2/HO-1 and NF-kappaB/ IkappaBalpha signaling pathway. Drug Des Dev Ther. 2018;12:1281-92.

\section{Publisher's Note}

Springer Nature remains neutral with regard to jurisdictional claims in published maps and institutional affiliations.

\footnotetext{
Ready to submit your research? Choose BMC and benefit from:

- fast, convenient online submission

- thorough peer review by experienced researchers in your field

- rapid publication on acceptance

- support for research data, including large and complex data types

- gold Open Access which fosters wider collaboration and increased citations

- maximum visibility for your research: over 100M website views per year
}

At BMC, research is always in progress.

Learn more biomedcentral.com/submissions 